\title{
VSC (Video Spectral Comparator)-6000 HS A Non- Destructive Technique for Identification of Blood
}

\author{
Chourasiya $\mathbf{S}^{1 *}$, Shaikh $\mathbf{T}^{2}$ and Dedhia $\mathbf{H}^{2}$ \\ ${ }^{1}$ Department of Forensic Science, Guru Ghasidas \\ Vishwavidyalaya, India \\ ${ }^{2}$ Department of Forensic Science, Institute of Forensic \\ Science, India \\ *Corresponding author: Shivam Chourasiya, \\ Department of Forensic Science, Guru Ghasidas \\ Vishwavidyalaya, Bilaspur, Chhattisgarh, India
}

Received: J anuary 16, 2017; Accepted: February 24 2017; Published: February 28, 2017

\begin{abstract}
Ascertaining a stain retrieved from a scene of crime to be blood or not, is a crucial aspect of a forensic investigation. Assuming and concluding the same requires several destructive presumptive and confirmatory assays. Moreover, if the retrieved quantity is scarce, then these destructive assays prove to be a gross discrepancy in forensic leads. This pilot study aims to promote the use of Visual Spectral Comparator (VSC) as a non-destructive presumptive technique to analyze the questioned stain. The technique is not definite but indicative and is based on the unique spectral characteristics of blood. Simultaneously the study also proposes that the use of porous material as the platform for the collection of blood yields comparatively good results vis-a-vis non porous methods as used in the above experimental study. The results were concluded on the basis of 25 blood samples collected on porous and non- porous surfaces from 25 healthy individual subjects and waste animal blood samples. Finding suggests that it can be an instrument of choice for routine analysis of blood samples in laboratories.
\end{abstract}

Keywords: Video spectral comparator; Blood; Nondestructive; Presumptive test; Whatman filter paper; Slide

\section{Introduction}

The Video Spectral Comparator 6000 (VSC) is a machine by Foster + Freeman. The machine utilizes many functions and different light sources to examine documents, and is usually used as a forensic tool to check the validity of valuable documents. Machine is a comparator, and it is also used to compare one set of images or spectra up against another. The VSC has also had a variety of uses in the cultural heritage sector, like analyzing ink and looking for deterioration in documents. As the name suggests the VSC is a comparator, and works by enhancing a document from different angles and in different light as well as taking spectral measurements. The results obtained by using the VSC are only indicative and not definite. The reason for this is that the machine is made to compare one set of data to another, and is not an analyzing instrument. But perhaps its results are good enough. The VSC in itself is costly, but the cost of use is not huge. The main item of expenditure is the time and cost of the user. Other than that it is mainly electricity and maintenance of the machine which decide its cost. The VSC also has another advantage compared to some of its competitors. It is a machine with more than one function. This can make it a good investment, since one gets a multifunctional tool, which can perhaps replace other instruments used in a conservation laboratory. The VSC uses different light sources UV light, Visible light, Infrared light. VSC instrument is based on spectroscopic Methods [1-16]. Spectrophotometric methods for blood identification are the measurement of interactions between electromagnetic radiation and matter. Electromagnetic radiation, which includes light, has characteristics of waves and particles. The absorption of radiation by matter is a quantized process, in that a molecule will only absorb radiation of certain discrete frequencies. These frequencies are determined by well-defined spacing's of energy levels in the molecule under investigation. The absorption of photons of high energy cause large changes in the molecules and correlate to moving electrons to higher energies [11]. One of the most important components of blood is hemoglobin. This pigment transports 65 times more oxygen in the erythrocytes than plasma does, and it can be found in two forms, deoxygenated and oxygenated [13]. In UV visible

1) To use VSC as a non-destructive technique for the presumptive identification of blood.

2) To utilize VSC (Visual Spectral Comparator) as a source of visible source of light in the spectroscopic analysis of blood.

3) To postulate that the canopy of blood spectrum remains "similar" with the passage of time.

4) To compare porous and nonporous substrates for better spectroscopic analysis of the unknown blood samples.

\section{Materials and Methods}

The study involved the collection of waste animal blood and 25 healthy individual blood samples under highly hygienic and controlled conditions with individual informed consent on two different substrates, porous (Whatman) and nonporous (slide) surfaces. Before this prior approval from Institutional Ethics Committee of Institute of Forensic Science, Mumbai (M.H.) was obtained (IEC/ 031/ 2014) for conducting the study. Separate sterile lancets were used to collect blood from individuals whose fingers were sterilized prior to pricking with absolute alcohol and 1-2 drops of blood was collected each on a sequentially numbered Whatman paper and a glass slide respectively. The used lancets and cottons were safely disposed off. The collected blood specimens were stored at room temperature in a safe plastic box away from moisture and direct sunlight. A 

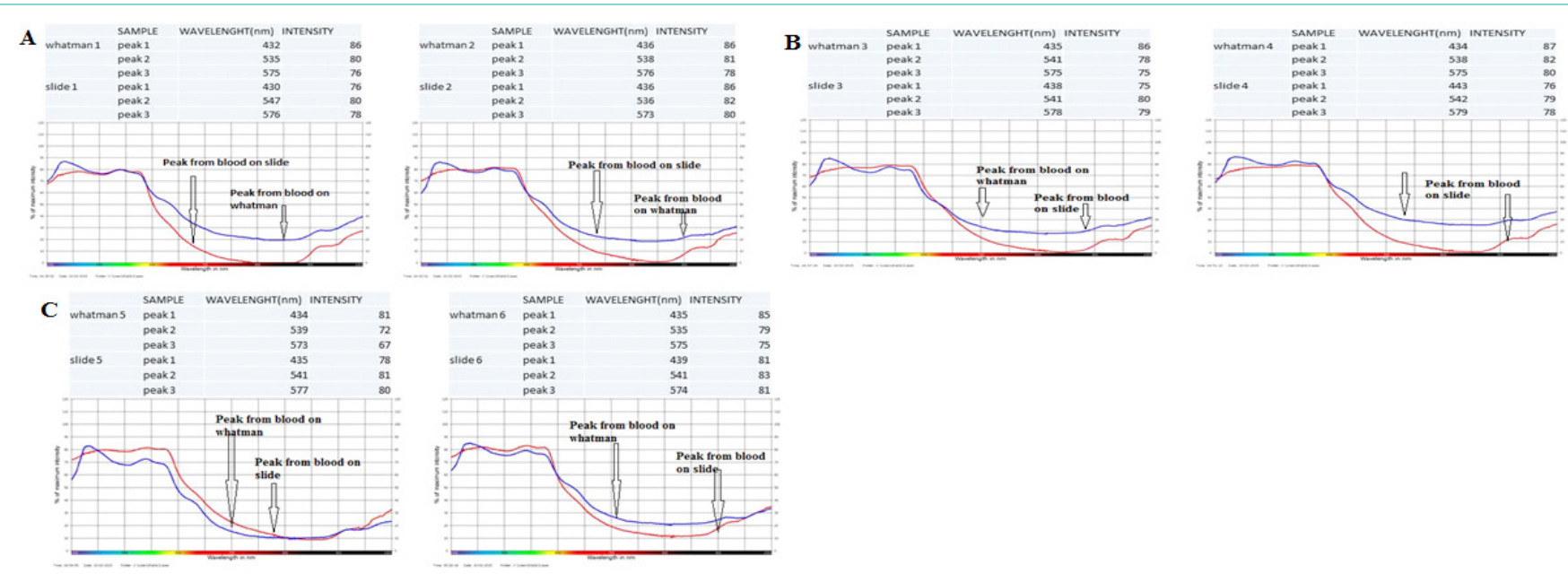

Figure 1: Showing results of Day 1.
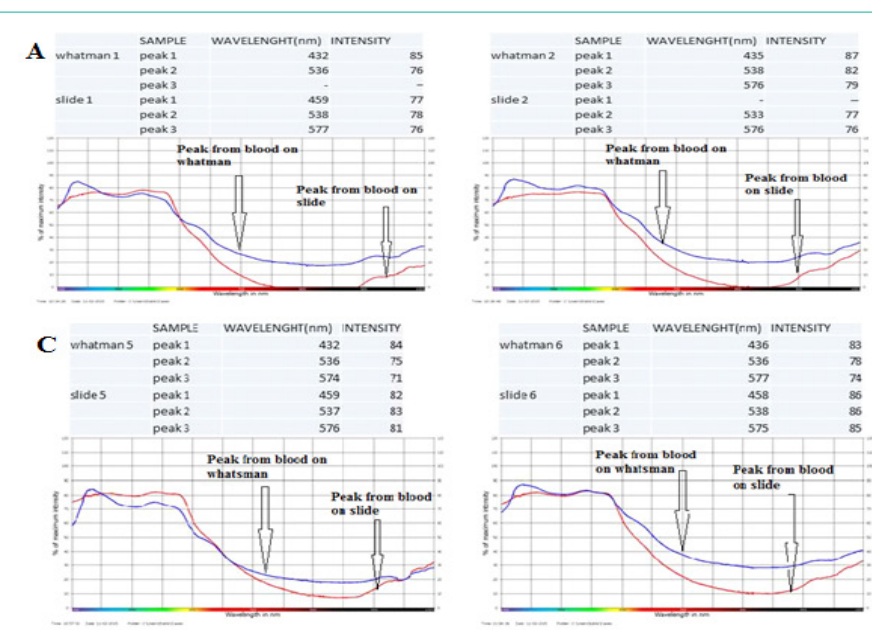
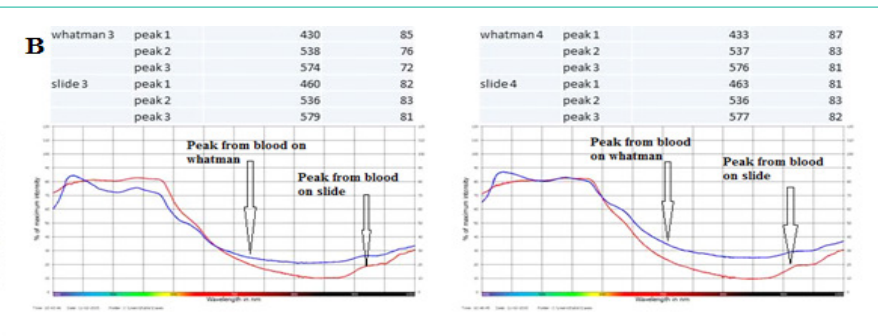

Figure 2: Showing the results of Day 2.

constant storage procedure was followed throughout the study. The analysis of the blood specimens, both Whatman and slide, was done using VSC 6000/HS Foster + Freeman. The procedure entailed the collection of spectral data of blood from both, Whatman and slide on a same graphical plane so as to enable clear comparison of their absorption characteristics on different platforms. In cases of both the types of samples, firstly, the scanning was initiated from UV light to visible light followed by measuring of the "white reference" i.e., the absorption spectra of the platform viz. the Whatman paper or the glass slides. Secondly, the absorption spectra of the blood specimen were collected, from which the previously recorded "white reference" was subtracted. This gave the absorption spectra of the actual blood sample at visible wavelength of light and graphical representation were developed using the inbuilt VSC 6000/ HS PC Suite software of the instrument. The graphical data and with their peaks were recorded and the graphs were saved as a bitmap file format on the computer drive. The very same procedures were strictly followed for all the 25 samples on different days. The samples were observed for the variability in the spectra at the interval of 24 hours, 48 hours, $4^{\text {th }}$ day, $6^{\text {th }}$ day, $8^{\text {th }}$ day, $15^{\text {th }}$ day. The spectral data was observed, recorded and analyzed for the spectral variations with the passage of age.

\section{Results and Discussion}

The study which aimed at the spectroscopic analysis of blood showed that VSC 6000/HS can be effectively used as a potential source for visible light. The (Figure 1-6) shows visible range of light from VSC can successfully be used for non-destructive and presumptive identification of blood. The spectra were found to be unique and characteristic to blood and dissimilar from the negative controls included in the experiment (Table 1 and 2). All of the 25 blood samples under study yielded a similar spectrum which entailed peaks characteristic to the constituents of blood (Figure 1-6). The samples continued to give similar spectrums up to a period of twelve months (Figure 7) after which, slight variations were witnessed with the gross appearances and characteristics remaining the same (Figure 7). These variations seen were probably due to ageing and decomposition of blood samples which were, although, stored under controlled conditions. A few extra peaks were witnessed in case of blood preserved using EDTA. This was due to the fact that EDTA itself has absorption peaks in the UV-Vis range (Figure 7). The spectrum 


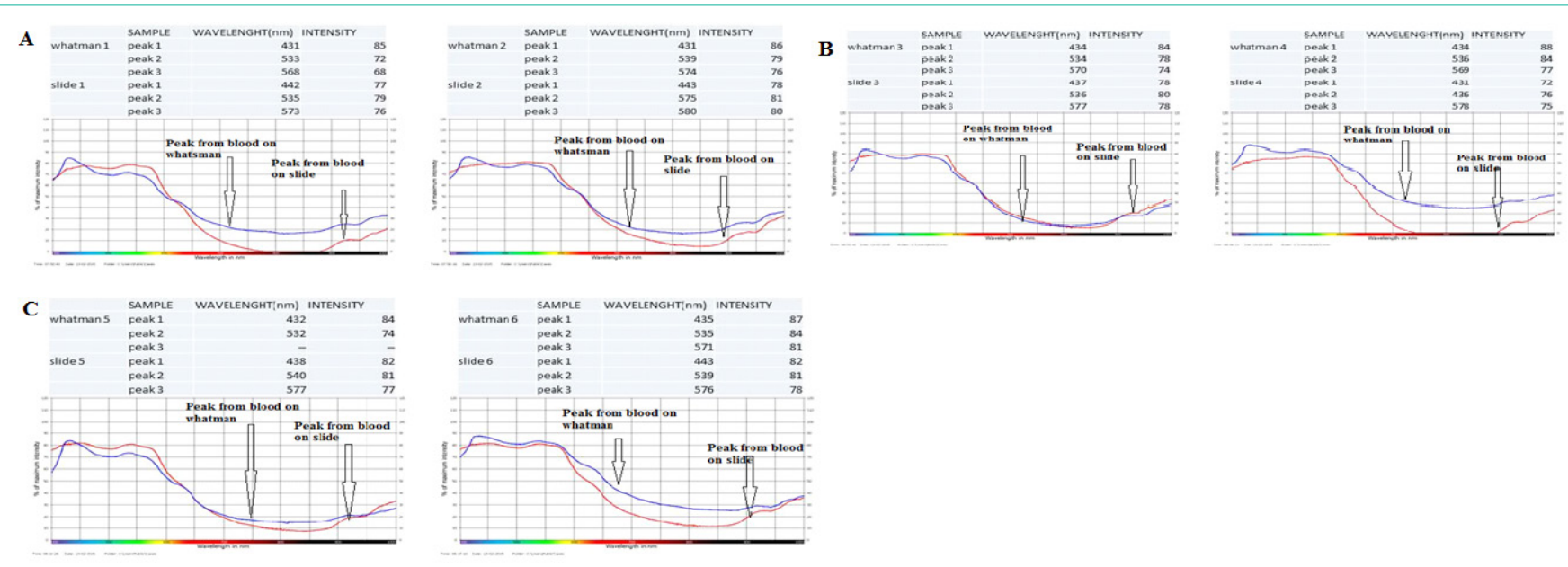

Figure 3: Showing results of Day 4.

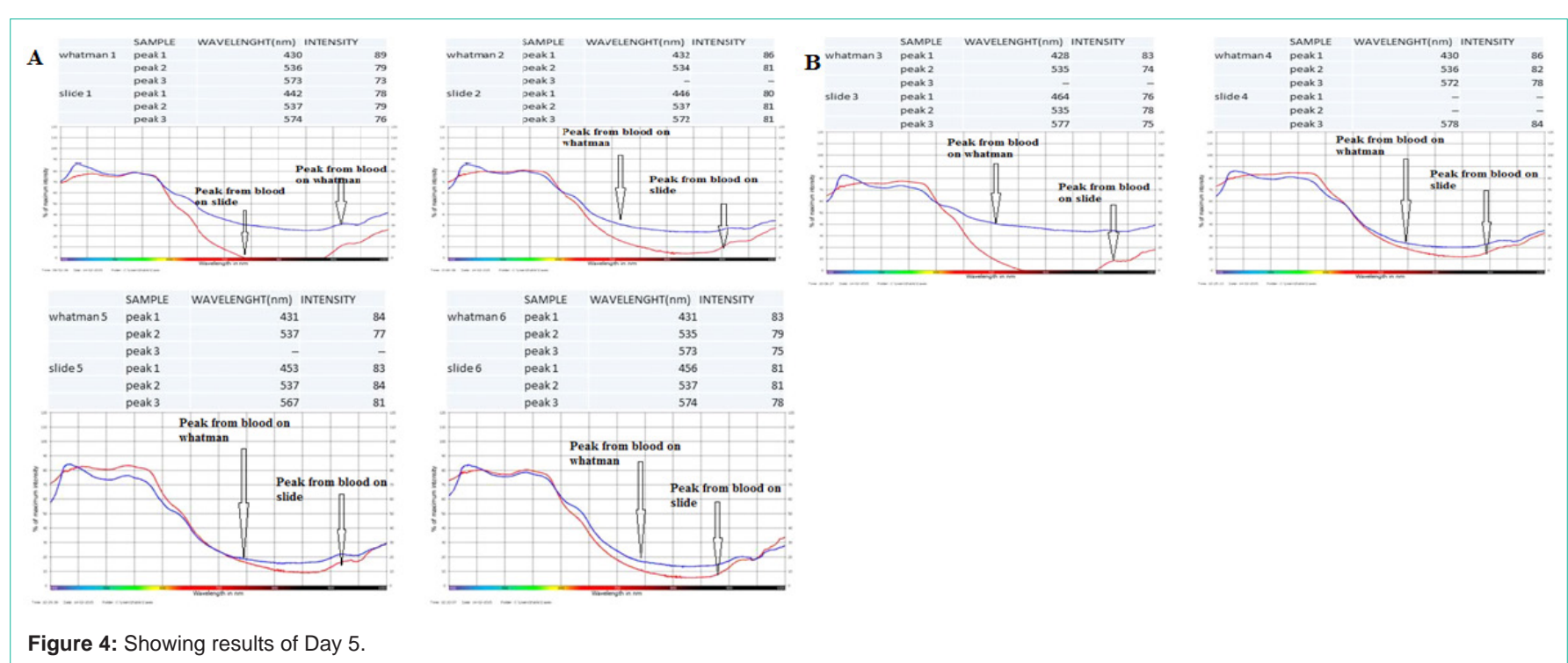

Figure 4: Showing results of Day 5

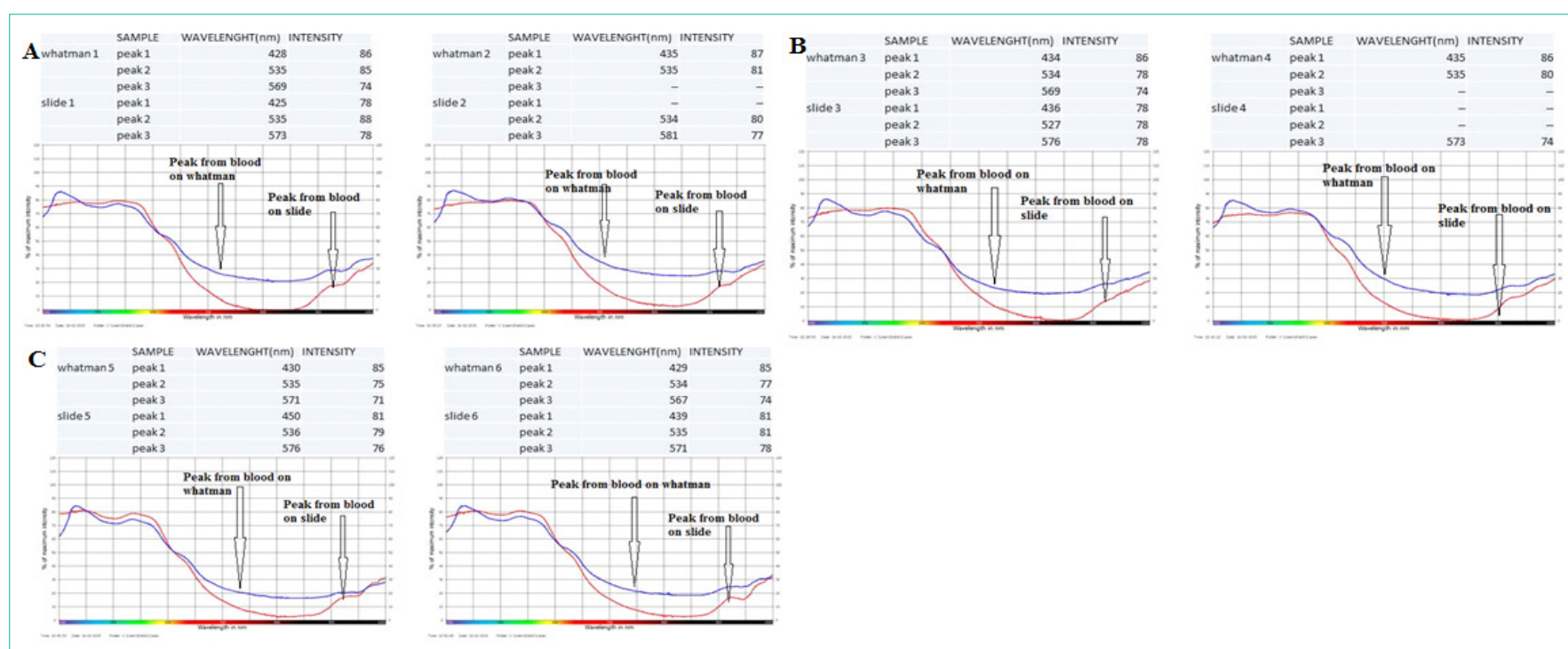

Figure 5: Showing results of Day 8. 

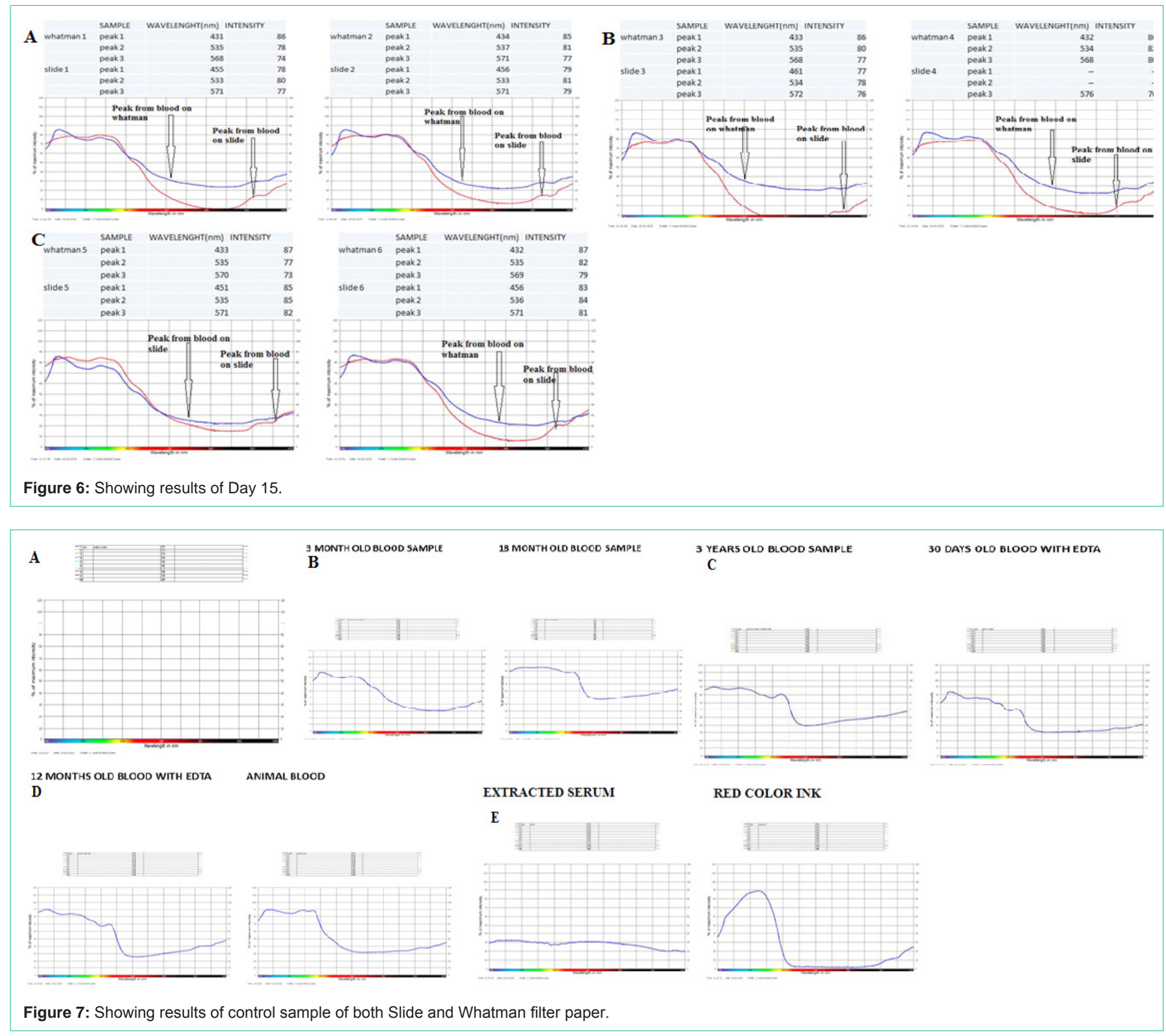

of animal blood was found to be very similar to that of human blood and thus the technique can be of no use in determination of origin of species (Figure 7). It was also evident from this pilot study that porous substrates used in the experiment gives distinct and better results as compared to non-porous substrates. (Table 1 and 2), The final negative control, i.e. spectrum of substrate Slide and Whatman filter paper produced no peaks, thus confirming the validity of the study (Figure 7).

\section{Conclusion}

Blood is one of the most crucial evidence available at the scene of crime. Its accurate identification and analysis is a must for proving guilt or innocence. If very trace amounts of blood is available, then it becomes difficult for the analysis team to utilize destructive methodologies for trial and error analysis. This pilot study successfully concluded that a questioned stain at a scene of crime can
Table 1: Range of wavelength observed for blood sample on porous surface.

\begin{tabular}{|c|c|c|}
\hline DAY & SAMPLE (WHATMANN FILTER PAPER) & WAVELENGTH(NM) \\
\hline 1 & - & 432 TO 575 \\
\hline 2 & - & 432 TO 577 \\
\hline 4 & - & 431 TO 571 \\
\hline 5 & - & 431 TO 573 \\
\hline 8 & - & 431 TO 571 \\
\hline 15 & - & 431 TO 571 \\
\hline
\end{tabular}

be subjected to non-destructive spectral analysis using VSC (Visual Spectral Comparator) and can be ascertained to be blood or not based on the very same spectral characteristics of blood. Thus, the non-destructively presumed stain to be blood can now be directed for a routine confirmatory assay which would not have been possible with a destructive presumptive technique. Moreover, the study also 
Table 2: Range of wavelength observed for blood sample on non porous surface.

\begin{tabular}{|c|c|l|c|}
\hline S.NO. & DAY & SAMPLE (GLASS SLIDE) & WAVELENGTH(NM) \\
\hline 1 & 1 & - & 432 TO 579 \\
\hline 2 & 2 & - & 458 TO 576 \\
\hline 3 & 4 & - & 431 TO 577 \\
\hline 4 & 5 & - & 459 TO 578 \\
\hline 5 & 8 & & 436 TO 576 \\
\hline 6 & 15 & & 451 T0 576 \\
\hline
\end{tabular}

revealed that the overall canopy of the spectroscopic graphs were "identical" in one year old samples. In addition to this, the study also revealed that a porous substrate for retrieval of blood evidence tends to give comparatively better outputs for analysis as compared to non-porous techniques. The advantage of the study is that does not involve preparation of reagents and less time consuming as other techniques to analyze the blood sample and disadvantage is that it's high cost and not possible to carry the machine at the crime scene but once employed does not cause as much as the cost required to prepare reagents which could be easily transported. In a nut shell, the study suggests a new, quick and easy, non-destructive, presumptive instrumental technique for analysis of blood which would help in aiding forensic investigations involving insufficient and aged blood samples.

\section{Acknowledgment}

The authors would like to express earnest and deepest gratitude to various individuals and organizations where in it would not have been possible to complete my project. Their kind support and help made this project to get successfully completed. I would like to extend my sincere thanks to all of them.

\section{References}

1. Horecker BL. The absorption spectra of hemoglobin and its derivatives in the visible and near infrared region. 1942.

2. Hanson EK, Ballantyne J. A blue Spectral Shift of the Hemoglobin Soret Band
Correlates with the Age (Time Since Deposition) of Dried Bloodstains. PLoS One. 2010; 5: 12830 .

3. Ballantyne J. Determination of the Age (Time since Deposition) of a Biological Stain document number. 2009; 226811.

4. Virkler K, Lednev IK. Analysis of body fluids for forensic purposes: From laboratory testing to non-destructive rapid confirmatory identification at a crime scene. Forensic Sci Int. 2009; 188: 1-17.

5. Kind SS, Patterson D, Owen GW. Estimation of the age of dried blood stains by a Spectrophotometric method. Forensic Sci. 1972; 1: 27-54.

6. Marc Lamontagne, Edita Botonjic-Sehic, Chris W. Brown, Mary Tsaparikos. Forensic Application of Near-Infrared Spectroscopy: Aging of Bloodstains. Spectroscopy. 2009; 24.

7. MartheAambø, Jonny Bjurman. Use of the Video Spectral Comparator 6000 as a non-destructive method for pigment identification ISSN. 2011; 11013303.

8. Gaensslen RE. Analysis of Biological Evidence. Wecht $\mathrm{CH}$ (editor). Forensic Sciences; 2000. 1.

9. Bremmer RH, Edelman G, Vegter TD, Bijvoets T, Aalders MC. Remote Spectroscopic Identication of Bloodstains. J Forensic Sci. 2011; 56: 14711475.

10. Scott Prahl. Oregon Medical Laser Center. Optical Absorption of Hemoglobin. 1999.

11. Lowndes S. Blood interference in fluorescence spectrum - Experiment, analysis and comparison with intra-operative measurements on brain tumor Linkoping University, Institute of technology. 2010.

12. Yodh AG, Chance B. Spectroscopy and Imaging with Diffusing Light. Physics Today.1995; 48: 34-40.

13. Tuan Vo-Dinh. Biomedical Photonics Handbook. 2003; 2.50-2.58.

14. Anderson S, Howard B, Hobbs GR, Bishop CP. A method for determining the age of a bloodstain. Forensic Sci Int. 2005; 148: 37-45.

15. Andrasko J. The estimation of age of bloodstains by HPLC analysis. J Forensic Sci. 1997; 42: 601-607.

16. Marthe Aambø. Use of the"Video Spectral Comparator 6000" as a nondestructive method for pigment identification (ISSN 1101-3303). 2011.
Austin J Forensic Sci Criminol - Volume 4 Issue 1 - 2017 ISSN : 2380-0801 | www.austinpublishinggroup.com Chourasiya et al. (C) All rights are reserved
Citation: Chourasiya S, Shaikh T and Dedhia H. VSC (Video Spectral Comparator)-6000 HS A Non-Destructive Technique for Identification of Blood. Austin J Forensic Sci Criminol. 2017; 4(1): 1057. 